\title{
NSABP FB-7: a phase II randomized neoadjuvant trial with paclitaxel + trastuzumab and/or neratinib followed by chemotherapy and postoperative trastuzumab in HER2 ${ }^{+}$breast cancer
}

Samuel A. Jacobs ${ }^{1 *}$, André Robidoux ${ }^{1,2}$, Jame Abraham ${ }^{1,3}$, José Manuel Pérez-Garcia ${ }^{4,5}$, Nicla La Verde ${ }^{6,7}$, James M. Orcutt ${ }^{1,8}$, Marina E. Cazzaniga ${ }^{5,9}$, Fanny Piette ${ }^{10}$, Silvia Antolín ${ }^{11}$, Elena Aguirre ${ }^{5}$, Javier Cortes ${ }^{4,5}$, Antonio Llombart-Cussac ${ }^{5}$, Serena Di Cosimo ${ }^{5,12}$, Rim S. Kim¹, Huichen Feng ${ }^{1}$, Corey Lipchik', Peter C. Lucas ${ }^{1,13}$, Ashok Srinivasan', Ying Wang ', Nan Song ', Patrick G. Gavin', April D. Balousek', Soonmyung Paik ${ }^{1,14}$, Carmen J. Allegra ${ }^{15}$, Norman Wolmark ${ }^{1,16}$ and Katherine L. Pogue-Geile ${ }^{1}$

\begin{abstract}
Purpose: The primary aim of NSABP FB-7 was to determine the pathologic complete response ( $\mathrm{pCR}$ ) rate in locally advanced HER2-positive (HER2 ${ }^{+}$) breast cancer patients treated with neoadjuvant trastuzumab or neratinib or the combination and weekly paclitaxel followed by standard doxorubicin plus cyclophosphamide. The secondary aims include biomarker analyses.

Experimental design: $p C R$ was tested for association with treatment, gene expression, and a single nucleotide polymorphism (SNP) in the Fc fragment of the IgG receptor Illa-158V/F (FCGR3A). Pre-treatment biopsies and residual tumors were also compared to identify molecular changes.
\end{abstract}

Results: The numerical pCR rate in the trastuzumab plus neratinib arm $(50 \%[95 \% \mathrm{Cl} 34-66 \%])$ was greater than that for single-targeted therapies with trastuzumab $(38 \%$ [95\% Cl 24-54]) or neratinib $(33 \%$ [95\% Cl 20-50]) in the overall cohort but was not statistically significant. Hormone receptor-negative $\left(\mathrm{HR}^{-}\right)$tumors had a higher $\mathrm{pCR}$ rate than $\mathrm{HR}^{+}$tumors in all three treatment arms, with the highest $\mathrm{PCR}$ rate in the combination arm. Diarrhea was the most frequent adverse event and occurred in virtually all patients who received neratinib-based therapy. Grade 3 diarrhea was reported in $31 \%$ of patients; there were no grade 4 events. Our 8-gene signature, previously validated for trastuzumab benefit in two different clinical trials in the adjuvant setting, was correlated with pCR across all arms of NSABP FB-7. Specifically, patients predicted to receive no trastuzumab benefit had a significantly lower pCR rate than did patients predicted to receive the most benefit $(P=0.03)$. FCGR genotyping showed that patients who were homozygous for the FC low-binding phenylalanine (F) allele for FCGR3A-158V/F were less likely to achieve pCR.

\footnotetext{
* Correspondence: samuel.jacobs@nsabp.org

${ }^{1}$ NSABP Foundation, Inc., Nova Tower 2, Two Allegheny Center - Ste 1200,

Pittsburgh, PA 15212, USA

Full list of author information is available at the end of the article
}

(c) The Author(s). 2019 Open Access This article is distributed under the terms of the Creative Commons Attribution 4.0 International License (http://creativecommons.org/licenses/by/4.0/), which permits unrestricted use, distribution, and reproduction in any medium, provided you give appropriate credit to the original author(s) and the source, provide a link to the Creative Commons license, and indicate if changes were made. The Creative Commons Public Domain Dedication waiver (http://creativecommons.org/publicdomain/zero/1.0/) applies to the data made available in this article, unless otherwise stated. 
(Continued from previous page)

Conclusions: Combining trastuzumab plus neratinib with paclitaxel increased the absolute pCR rate in the overall cohort and in $\mathrm{HR}^{-}$patients. The 8-gene signature, which is validated for predicting trastuzumab benefit in the adjuvant setting, was associated with $\mathrm{PCR}$ in the neoadjuvant setting, but remains to be validated as a predictive marker in a larger neoadjuvant clinical trial. HR status, and the FCGR3A-158V/F genotype, also warrant further investigation to identify HER2 ${ }^{+}$patients who may benefit from additional anti-HER2 therapies beyond trastuzumab. All of these markers will require further validation in the neoadjuvant setting.

Trials registration: ClinicalTrials.gov, NCT01008150. Retrospectively registered on October 5, 2010.

Keywords: Breast cancer, Neoadjuvant, Neratinib

\section{Introduction}

Human epidermal growth factor receptor 2 (HER2/ $E R B B 2$ ) is overexpressed in approximately $25 \%$ of breast cancers and drives hyperactivation of the HER2 pathway via downstream signaling initiated by receptor homo- or hetero-dimerization with other HER family members (HER2/HER2, HER2/EGFR, HER2/HER3). Prior to the advent of HER2-targeted therapeutics, HER2-positive disease $\left(\mathrm{HER}_{2}{ }^{+}\right.$) was associated with a poor clinical outcome, but this dramatically changed with the development of trastuzumab and additional HER2-specific agents, especially in early HER2 ${ }^{+}$breast cancer [1-5]. Unfortunately, the vast majority of patients with metastatic HER2 ${ }^{+}$disease who initially respond to trastuzumab will develop resistance [6]. This has stimulated substantial interest in understanding both the mechanisms of resistance and development of additional anti-HER2 agents.

Small-molecule tyrosine kinase inhibitors (TKIs) suppress the growth of $\mathrm{HER}^{+}$breast cancer cells in vitro and tumor xenografts in vivo by inhibiting phosphorylation of $E R B B$ receptor tyrosine kinases (RTKs) and thus preventing activation of downstream signaling mediators [7-9]. Lapatinib, a reversible inhibitor of EGFR and HER2 RTKs, is active in the first and subsequent lines of treatment of patients with HER2 $^{+}$metastatic disease and has received US Food and Drug Administration (FDA) approval when combined with capecitabine in patients with progressive disease after anthracycline, a taxane, and trastuzumab [10]. In two neoadjuvant trials, NeoALTTO $(N=455)$ [11] and CALGB $40601(N=305)$ [12], the combination of lapatinib, trastuzumab, and paclitaxel was compared to paclitaxel combined with either antiHER2 agent alone. The rate of pathologic complete response ( $\mathrm{pCR})$, defined as no residual invasive disease (in the breast), was numerically higher in patients receiving lapatinib and trastuzumab than in patients receiving trastuzumab alone (NeoALTTO $51 \%$ vs $29 \%, P=0.0001$; CALGB $56 \%$ vs $46 \%, P=$ 0.13 ). In both studies, a statistically significant increase in $\mathrm{pCR}$ was observed in patients with $\mathrm{HR}^{-}$ disease treated with dual anti-HER2 therapy compared to single-agent anti-HER2 therapy.

Neratinib, an irreversible inhibitor of pan-ERBB RTKs, is more potent than lapatinib in HER2 ${ }^{+}$breast cancer cell lines and in human tumor xenografts [13]. In phase II studies with neratinib monotherapy in patients with HER2 $^{+}$breast cancer, the objective response rate was $24 \%$ in trastuzumab-refractory patients and $56 \%$ in trastuzumab-naive patients [14].

Clinical data in $\mathrm{HER}^{+}$metastatic breast cancer patients treated with neratinib at $240 \mathrm{mg} /$ day and paclitaxel at $80 \mathrm{mg} / \mathrm{m}^{2}$ on days 1,8 , and 15 of a 28 -day cycle resulted in a median progression-free survival of 47.9 weeks and an objective response rate of $71 \%$ with responses observed in patients with prior trastuzumab, lapatinib, and taxane therapy [15]. The combination of weekly paclitaxel with trastuzumab plus neratinib in women with metastatic, HER2 ${ }^{+}$advanced breast cancer was evaluated in the phase Ib NSABP FB-8 study (NCT01423123). The recommended phase II dose of neratinib was $200 \mathrm{mg} /$ day. Impressive clinical activity was observed in these heavily pre-treated patients with an objective response rate of $38 \%$ and a clinical benefit rate of $52 \%$ [16].

Taken together, these studies suggest that combining non-cross resistant anti-HER2 therapy may result in a higher pCR rate. Here, we report the safety and efficacy of a randomized study in patients with locally advanced, HER $2^{+}$breast cancer treated in the neoadjuvant setting with trastuzumab, neratinib, or the combination, in each case administered for 16 weeks with paclitaxel followed by standard chemotherapy with doxorubicin plus cyclophosphamide (AC) for 4 cycles.

\section{Methods}

\section{Patients and study design}

This phase II trial, which opened for accrual in the US and Canadian NSABP Foundation sites, was designed originally as a two-arm study with 2:1 randomization to evaluate trastuzumab or neratinib with paclitaxel followed by AC. In December 2011, after 30 patients were enrolled, accrual was placed on hold. The decision to hold accrual 
was based on reports that dual anti-HER2 inhibition with trastuzumab plus lapatinib or trastuzumab plus pertuzumab increased the $\mathrm{pCR}$ rate in neoadjuvant breast cancer [11, 17]. From May 2011 to July 2012, the NSABP Foundation conducted a phase I dose-escalation study in patients with $\mathrm{HER}^{+}$metastatic disease evaluating the combination of trastuzumab, neratinib, and paclitaxel. This study established the recommended phase II dose of neratinib as $200 \mathrm{mg} /$ day in combination with trastuzumab and paclitaxel [16]. In August 2012, FB-7 reopened as a randomized (1:1:1) study adding the third arm of trastuzumab, neratinib, and paclitaxel followed by AC. The randomization was adjusted so that at the completion of the study, the three arms, would have an equal number of evaluable patients (total study $N=126$ ). On September 30, 2013, the US FDA granted accelerated approval to pertuzumab in combination with trastuzumab and docetaxel for use in the neoadjuvant setting in women with HER2 ${ }^{+}$ breast cancer based on improved pCR in the NeoSphere study (pertuzumab, trastuzumab, docetaxel, pCR rate 45.8\%) [17] and TRYPHAENA study (pertuzumab, trastuzumab, carboplatin, docetaxel, pCR rate 66.2\%) [18].

On October 22, 2013, the NSABP closed arms 1 and 2 of FB-7 to additional accrual in the USA. From October 22, 2013, to April 2014, arm 3 remained open as a nonrandomized arm to accrue an additional 12 patients in order to obtain more robust safety information on US patients. These patients are included only in the biomarker analysis with the exception of the MammaPrint ${ }^{\circ}$ analysis, which included only randomized patients. European sites, in which dual anti-HER2 therapy was not the standard of care, were opened to complete the accrual to the randomized three-arm trial (total study $N=126$ ). Canadian sites continued to accrue to the three-arm trial. The last patient enrolled on November 12, 2014.

Eligible patients included women $\geq 18$ years with ECOG PS of $0-1$, invasive adenocarcinoma of the breast, $\mathrm{HR}^{+}$or $\mathrm{HR}^{-}$disease, and HER2 positivity defined as a score of $3+$ by immunohistochemistry (IHC), or evidence of gene amplification by fluorescence in situ hybridization (FISH) or chromogenic in situ hybridization (CISH). HER2 testing was performed locally without central confirmation. We are not able to provide HER2 status based upon the guidelines published in 2018 [19].

Patients with AJCC stage IIB, IIIA, IIIB, or IIIC were eligible. Patients were required to have the following baseline laboratory studies: an absolute neutrophil count of $\geq 1000 / \mathrm{mm}^{3}$, platelet count of $\geq 100,000 / \mathrm{mm}^{3}$, hemoglobin of $\geq 9 \mathrm{~g} / \mathrm{dL}$, serum creatinine $\leq 1.5 \times$ the upper limits of normal (ULN), total bilirubin of $\leq 1.5 \times$ the ULN, AST, and ALT of $\leq 1.5 \times$ ULN. Left ventricular ejection fraction (LVEF) $\geq 50 \%$ assessed by either $2 \mathrm{D}$ echocardiogram or MUGA scan was required. The first
60 patients were required to have a research biopsy before therapy initiation; this was subsequently dropped as a requirement.

Patients were excluded if they had any evidence of metastatic disease, active hepatitis $\mathrm{B}$ or $\mathrm{C}$ with abnormal liver function tests, intrinsic lung disease causing dyspnea, persistent $\geq$ grade 2 diarrhea, sensory-motor neuropathy $\geq$ grade 2 , conditions that would prohibit intermittent administration of corticosteroids for paclitaxel premedication, or active cardiac disease including recent myocardial infarction, symptomatic arrhythmia, or angina pectoris.

The study protocol was approved by the Institutional Review Boards of each participating institution, and all patients provided written informed consent. The study was conducted according to the Good Clinical Practice and the Declaration of Helsinki and its amendments. The authors had full control of all primary data. The datasets during and/or analyzed during the current study will be available from the corresponding author on reasonable request and with permission from PUMA Biotechnology.

Patients in arm 1 (control) received 4 cycles of paclitaxel $80 \mathrm{mg} / \mathrm{m}^{2}$ administered on days 1,8 , and 15 of a 28-day cycle with trastuzumab $4 \mathrm{mg} / \mathrm{kg}$ loading dose, then $2 \mathrm{mg} / \mathrm{kg}$ weekly for a total of 16 doses. Following paclitaxel and trastuzumab, doxorubicin (A) $60 \mathrm{mg} / \mathrm{m}^{2}$ and cyclophosphamide (C) $600 \mathrm{mg} / \mathrm{m}^{2}$ were given every 3 weeks for 4 cycles. In arm 2 (experimental), in place of trastuzumab, patients received neratinib $240 \mathrm{mg}$ taken orally once daily beginning on day 1 of paclitaxel and continuing through day 28 of the final cycle of paclitaxel. In arm 3 (experimental), both trastuzumab and neratinib were given with paclitaxel as described above; however, neratinib was administered at $200 \mathrm{mg} /$ day. After recovery from all chemotherapy, patients had their definitive surgery and completion of 1 year of trastuzumab. The decisions regarding hormonal therapy and radiotherapy were at the discretion of the treating physician.

Standard pre-medications were given before each paclitaxel administration and during AC. Because diarrhea is expected with neratinib, early in the study, diarrhea management was initiated after the first diarrheal stool with loperamide $4 \mathrm{mg}$ and then $2 \mathrm{mg}$ after each loose stool thereafter. Despite this management plan, diarrhea was still consistently noted within 2 weeks of study therapy. Subsequently, diarrheal guidelines were amended to mandate primary prophylaxis with loperamide beginning with the first dose of therapy. At all treating sites, patients were contacted at 24,48 , and $72 \mathrm{~h}$ during the first week of treatment on the neratinib-containing arms to reenforce anti-diarrheal management.

The primary endpoint was $\mathrm{pCR}$, defined as no residual invasive disease in the breast and lymph nodes $(y \mathrm{pT} 0 /$ 
N0). Randomly assigned patients who received any protocol therapy were included in the analysis. Secondary endpoints included clinical complete response, pCR in the breast, and rate of adverse events (AEs). Due to the small sample size and a limited number of tissue samples available, all molecular correlates are exploratory.

\section{Study assessments}

Safety was assessed by physical examination, interim history, and laboratory assessment. AE assessment occurred on days 1 and 15 of cycle 1 and on day 1 of each subsequent cycle, and 2 to 4 weeks after the last cycle of AC. $\mathrm{AE}$ reporting was assessed according to the National Cancer Institute Common Terminology Criteria for Adverse Events (NCI CTCAE) version 3.0. AEs occurring at the US or Canadian sites were continuously monitored and reviewed by the NSABP medical review team. European sites were monitored by MedSIR every 6 to 10 weeks.

\section{Molecular analyses \\ Tumor RNA extraction}

RNA was isolated from pre-treatment tumor biopsies and residual disease utilizing one to five $5-\mu \mathrm{m}$ tissue sections depending on the size of the tumor area within a slide. Separate tissue sections were required for RNA and DNA. Extractions were prepared using the AllPrep DNA/RNA FFPE kit from Qiagen and following the manufacturer's recommendations.

\section{FCGR genotyping}

Whole blood was collected in ACD tubes from patients $(n=80)$ before treatment. Peripheral blood monocytes (PBMC) were isolated and stored at $-80^{\circ} \mathrm{C}$. Genotyping of rs1801274 (FCGR2A-131R/H) and rs396991 (FCGR3A$158 \mathrm{~V} / \mathrm{F}$ ) was performed as previously described [20].

\section{RNA-Seq}

Details of the preparation, performance, and analysis of the RNA-Seq libraries are included in Additional file 1: Methods.

\section{MammaPrint scoring}

The proprietary MammaPrint 70-gene Breast Cancer Recurrence Assay was performed at Agendia, using 50 ng of RNA for all available patients with pre-treatment biopsies with sufficient RNA $(n=45)$; one case failed RNA and hybridization quality control, and in five cases, pCR information was missing. MammaPrint was performed retrospectively to determine the number of lowrisk patients enrolled in the study.

\section{Statistical analysis}

This is a non-comparative, randomized phase II study in which patients with $\mathrm{HER}^{+}$, locally advanced disease were randomized to one of the three arms. The study was designed originally as a two-arm trial with 2:1 randomization. However, when it became apparent that dual anti-HER2 regimens showed early favorable results, the trastuzumab plus neratinib arm was added and the randomization balanced among arms. The primary endpoint was $\mathrm{PCR}$, defined as no evidence of invasive disease in the breast and nodes ( $y \mathrm{pT} 0 / \mathrm{N} 0)$. We determined that a sample size per arm of 42 patients would offer $80 \%$ power to test the null hypothesis that the response rate would be $30 \%$ or less at a one-sided alpha of 0.05 . pCR was analyzed descriptively by treatment arm and, for exploratory purposes, was compared across the three arms (two-by-two comparisons) using the stratified Cochran-Manel-Haenszel statistic (two-sided $P$ values). The analyses were stratified in such a way that patients randomly assigned to the study prior to the addition of arm 3 were considered in a separate stratum from those randomly assigned after the addition of arm 3 .

Secondary aims and exploratory molecular analyses were performed for exploratory purposes with Fisher's exact or chi-square tests, not adjusted for multiplicity. Any comparison that reached nominal statistical significance should be interpreted with due regard to the multiple comparisons performed, the small number of patients in the subgroups, and the fact that some analyses were performed retrospectively.

\section{Results}

\section{Patient characteristics}

There were 126 patients enrolled in the randomized portion of this study who received at least one dose of study drug and are included in Table 1. Distribution of tumor stage was similar across arms, as were tumor size and method of determination of HER2 status. There were more postmenopausal patients in arm 2 and fewer $\mathrm{HR}^{+}$ patients in arm 3 although this difference was not statistically significant. Three patients withdrew from the study before receiving any study drug and were replaced (Fig. 1 CONSORT Diagram: NSABP FB-7).

\section{Adverse events}

The most common grade 1-4 treatment-emergent AEs that occurred with anti-HER2 therapy plus paclitaxel are shown in Table 2. Diarrhea was the most frequent $\mathrm{AE}$ and occurred in virtually all patients who received neratinib-based therapy, with grade 3 diarrhea reported in $31 \%$ of patients; there were no grade 4 diarrheal events. During the course of the study, primary antidiarrheal prophylaxis was instituted and resulted in a decrease in grade 3 diarrhea in the neratinib arms to $21 \%$. 
Table 1 NSABP FB-7: patient characteristics by randomized arm

\begin{tabular}{|c|c|c|c|}
\hline & Arm $1, n=42$ & Arm $2, n=42$ & $\operatorname{Arm} 3, n=42$ \\
\hline \multicolumn{4}{|l|}{ Age, years } \\
\hline Median & 50.0 & 56.0 & 50.0 \\
\hline Range & $33-71$ & $29-71$ & $31-77$ \\
\hline \multicolumn{4}{|c|}{ Performance status } \\
\hline ECOG 0 & $42(100 \%)$ & $42(100 \%)$ & $41(97.6 \%)$ \\
\hline ECOG 1 & 0 & 0 & $1(2.4 \%)$ \\
\hline \multicolumn{4}{|l|}{ Tumor stage } \\
\hline$\| \mathrm{B}$ & $18(42.8 \%)$ & $19(45.2 \%)$ & $20(47.6 \%)$ \\
\hline III & $21(50 \%)$ & $20(47.6 \%)$ & $22(52.3 \%)$ \\
\hline Missing & $3(7.1 \%)$ & $3(7.1 \%)$ & 0 \\
\hline Inflammatory & $4(9.5 \%)$ & $7(16.7 \%)$ & $4(9.5 \%)$ \\
\hline \multicolumn{4}{|c|}{ Menopausal status } \\
\hline Pre & $25(60 \%)$ & 16 (38\%) & $23(55 \%)$ \\
\hline Post & 17 (40\%) & $26(62 \%)$ & $19(45 \%)$ \\
\hline \multicolumn{4}{|c|}{ Hormone receptor } \\
\hline Positive & $28(67 \%)$ & 29 (69\%) & $23(55 \%)$ \\
\hline Negative & $14(33 \%)$ & 13 (31\%) & 19 (45\%) \\
\hline \multicolumn{4}{|c|}{ Clinical tumor size } \\
\hline Mean, cm & 4.50 & 5.04 & 4.72 \\
\hline Median, cm & 3.70 & 5.50 & 5.00 \\
\hline Range, cm & $0.0-18.0$ & $0.0-10.0$ & $0.0-9.0$ \\
\hline \multicolumn{4}{|l|}{ HER2 } \\
\hline $\mathrm{IHC}$ & $24(57.1 \%)$ & $24(57.1 \%)$ & $25(59.5 \%)$ \\
\hline ISH & 15 (35.7\%) & $12(28.6 \%)$ & $11(26.2 \%)$ \\
\hline $\mathrm{IHC}$ and ISH & 3 (7.1\%) & 6 (14.3\%) & 6 (14.3\%) \\
\hline
\end{tabular}

Grade 2 constipation occurred in 5\% and 2\% of arms 2 and 3 , respectively. Higher grades of constipation were not observed. Grade 3 transaminase elevation was observed in $7 \%$ of the patients in the neratinib-containing arms (Table 2), which is very similar to the findings in ISPY2 [21].

At least one neratinib dose reduction occurred in $38 \%$ of patients in arm 2 and 52\% of patients in arm 3. During paclitaxel plus anti-HER2 therapy, at least one drug was discontinued before completion of 4 cycles in $19 \%, 21 \%$, and $40 \%$ in arms 1,2 , and 3 , respectively. Dose intensity of the targeted agents calculated until the time of discontinuation is shown in Additional file 1: Table S1.

\section{Efficacy and compliance}

Clinical assessment in patients with a baseline palpable breast lesion reported an overall complete response in $70 \%$ of patients with $66 \%, 71 \%$, and $74 \%$ in arms 1,2 , and 3 , respectively. A total of $91 \%$ of patients had a definitive surgery equally distributed across arms. The pCR rates (the breast and nodes) were $38 \%, 33 \%$, and $50 \%$ in arms 1 ,
2 , and 3 , respectively (see Table 3 ). pCR rates in the breast were $50 \%, 38 \%$, and $52 \%$ in arms 1,2 , and 3 , respectively. There were 11 patients for whom we do not have surgical findings. Reasons include early progression $(n=3)$, consent withdrawal, or lost to follow-up $(n=8)$. All patients for whom data are missing were considered no pCR. At 2 years from the random assignment, recurrence had been reported in five patients ( $\operatorname{arm} 1=0, \operatorname{arm} 2=3$, and arm $3=2$ ). No deaths were reported.

\section{Assessment of breast cancer recurrence risk}

The I-SPY2 trial (19) excluded patients with low risk recurrence score based on the MammaPrint 70-gene breast cancer recurrence assay.

In FB-7, we did not use this assay to prospectively exclude low-risk patients, although we retrospectively assessed the risk of recurrence based on the MammaPrint score in 45 randomly assigned patients. Analysis of the available samples showed that there were only 3 patients $(5.8 \%)$ with a low-risk MammaPrint result (Additional file 1: Figure S1). No patient with a low-risk MammaPrint score had a pCR. There were 2 patients with low-risk disease in the trastuzumab arm and 1 in the neratinib arm. Thus, it is unlikely that the inclusion of patients at low risk was responsible for the discordant results between I-SPY2, which showed single-agent neratinib to be superior to trastuzumab in $\mathrm{HER}^{+}$and $\mathrm{HR}^{-}$ patients, whereas FB-7 showed no difference in pCR rates between single-agent neratinib and trastuzumab.

\section{Hormone receptor status and $\mathrm{pCR}$}

We tested the association of HR status with pCR $(N=$ 126). Similar to other neoadjuvant studies (Additional file 1 : Table S2), patients with $\mathrm{HR}^{+}$tumors were less likely to achieve pCR after treatment with anti-HER2 therapies than those patients who were $\mathrm{HR}^{-}$(Table 3). This difference in $\mathrm{pCR}$ rates between $\mathrm{HR}^{+}$and $\mathrm{HR}^{-}$patients reached statistical significance only within arm 3 (trastuzumab + neratinib + paclitaxel) (Table 3$)$. $\mathrm{HR}^{-}$patients who were treated with the trastuzumab plus neratinib combination had the highest numerical pCR rate $(73.7 \%)$, although this rate was not statistically different compared to $\mathrm{HR}^{-}$patients treated with trastuzumab $(57.1 \%, P=0.46)$.

\section{FCGR genotyping}

Antibody dependent cellular cytotoxicity (ADCC) is a mechanism to determine the therapeutic efficacy of monoclonal antibodies such as trastuzumab. ADCC is initiated when the Fcy receptor IIIa (FcyRIIIa) on NK cells binds to the Fc region of trastuzumab. FcyIIIaR has been shown to have a differential affinity to the Fc portion of IgG1 depending upon a SNP at position $158(\mathrm{~V} / \mathrm{F})$ in the FCGR3A gene. The FcyRIIIa-158V protein has a higher affinity for IgG1 antibodies than 


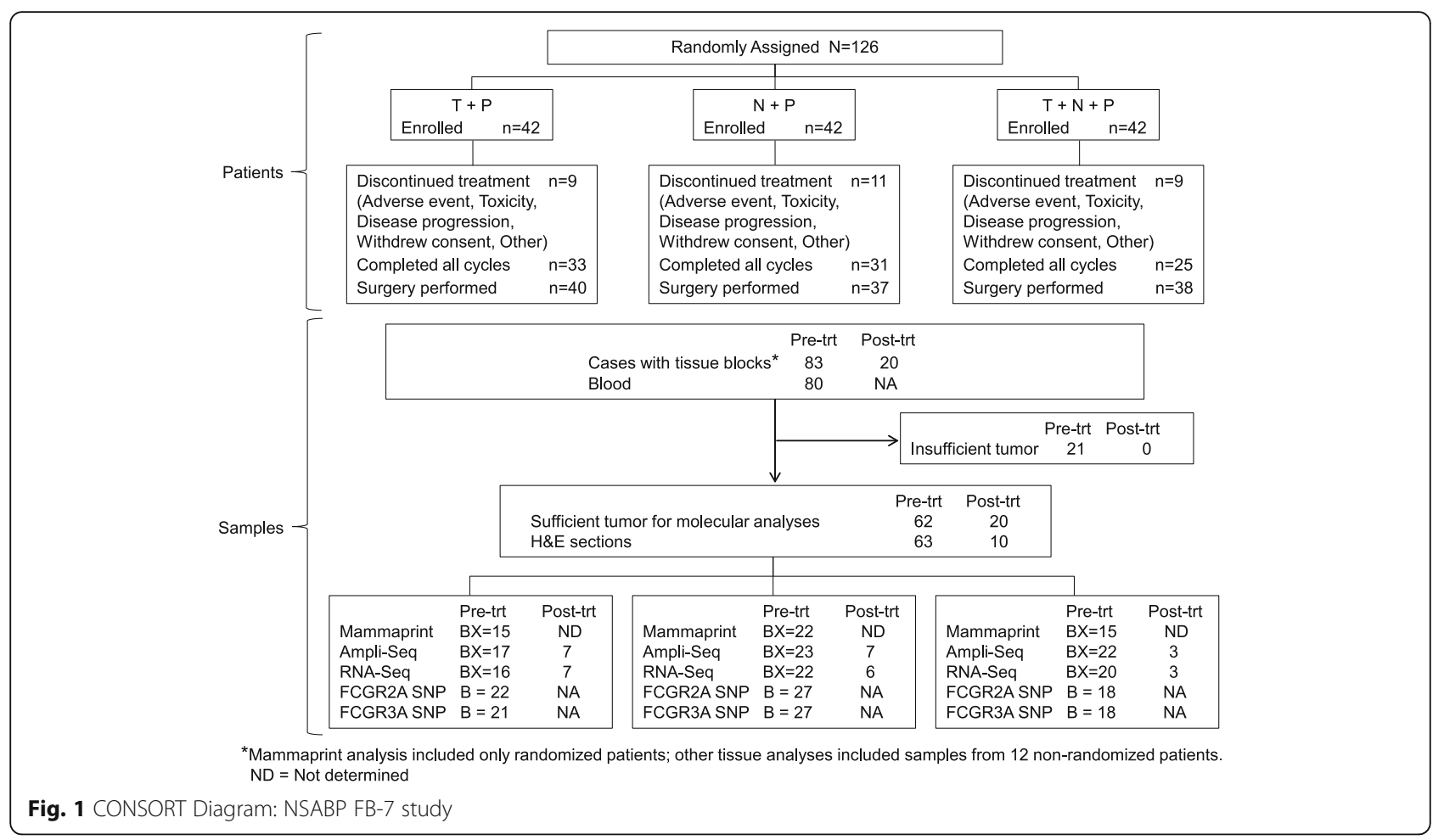

does the FcyRIIIa-158F protein. We have previously shown an association of the FCGR3A-158F/V genotypes with benefit from trastuzumab in the adjuvant setting [20]. FB-7 provided an opportunity to test for an association of the FCGR3A-158F/V genotypes with pCR in the neoadjuvant setting. The pCR rate for patients with an FCGR3A genotype with one or two of the high-binding alleles (FCGRA-158V) was 19/31 [61.3\%], in comparison with only $7 / 27$ [25.9\%] for patients who were homozygous for the low-binding allele (FCGRA-158F). The difference in $\mathrm{pCR}$ rates between low- $(\mathrm{F} / \mathrm{F})$ and high-binding alleles $(\mathrm{F} / \mathrm{V}$ or $\mathrm{V} / \mathrm{V})$ was statistically significant for all patients analyzed together $(P=0.009)$ and also for patients in the trastuzumab arm $(P=0.02)$, but not in the neratinib $(P=$ $0.4)$ or in the trastuzumab plus neratinib arms $(P=0.6)$, which would be predicted based on the mechanism of action of neratinib (Table 4). Because the therapeutic efficacy of neratinib is not dependent upon eliciting antibodydependent cellular cytotoxicity (ADCC) activity, we postulated that patients with the FCGR3A-158F/F genotype would have a better pCR rate in the trastuzumab plus neratinib arm than in the trastuzumab arm [1]. Indeed, we do see a numerically higher $\mathrm{pCR}$ rate in the trastuzumab plus neratinib arm $(3 / 7=43 \%)$ than in the trastuzumab arm $(1 /$ $8=13 \%)$.

\section{8-Gene trastuzumab benefit predictive signature correlates with $\mathrm{pCR}$}

We developed and validated an 8-gene expression signature that predicted trastuzumab benefit in the adjuvant setting in NSABP B-31 [22] and NCCTG9831

Table 2 NSABP FB-7: treatment-emergent adverse events during trastuzumab plus paclitaxel (arm 1), neratinib plus paclitaxel (arm 2), or trastuzumab plus neratinib plus paclitaxel (arm 3) (all cycles)

\begin{tabular}{|c|c|c|c|c|c|c|c|c|c|}
\hline \multirow[t]{2}{*}{ Event } & \multicolumn{3}{|c|}{ Arm $1, n=42$} & \multicolumn{3}{|c|}{ Arm 2, $n=42$} & \multicolumn{3}{|c|}{$\operatorname{Arm} 3, n=42$} \\
\hline & Grades 1-2 & Grade 3 & Grade 4 & Grades 1-2 & Grade 3 & Grade 4 & Grades 1-2 & Grade 3 & Grade 4 \\
\hline Diarrhea & $16(38 \%)$ & 0 & 0 & $29(69 \%)$ & $13(31 \%)$ & 0 & $28(66 \%)$ & $13(31 \%)$ & 0 \\
\hline Nausea & 12 (29\%) & 0 & 0 & 25 (60\%) & 0 & 0 & 19 (46\%) & $1(2 \%)$ & 0 \\
\hline Rash & $9(21 \%)$ & 0 & 0 & $9(21 \%)$ & 0 & 0 & $6(14 \%)$ & 0 & 0 \\
\hline Transaminase elevation & $9(21 \%)$ & $1(2 \%)$ & 0 & $16(38 \%)$ & $3(7 \%)$ & 0 & $18(43 \%)$ & $3(7 \%)$ & 0 \\
\hline Fatigue & $18(43 \%)$ & 0 & 0 & $21(50 \%)$ & $1(2 \%)$ & 0 & $17(40 \%)$ & $1(2 \%)$ & 0 \\
\hline Neuropathy & $15(36 \%)$ & 0 & 0 & $13(31 \%)$ & 0 & 0 & $6(14 \%)$ & $1(2 \%)$ & 0 \\
\hline
\end{tabular}


Table 3 NSABP FB-7: pathologic complete response ( $\mathrm{pCR}$ ) (breast and nodes) by treatment arm and HR status

\begin{tabular}{|c|c|c|c|c|c|c|c|}
\hline $\begin{array}{l}\text { Treatment arms, } \\
n=42 \text { each arm }\end{array}$ & Total pCR & $\begin{array}{l}P \text {-value for } \mathrm{pCR} \\
\mathrm{T} \text { vs Other Arms } \\
\text { in all patients }{ }^{\mathrm{a}}\end{array}$ & $\mathrm{pCR} \mathrm{HR^{+ }}$ & $\begin{array}{l}\text { P-value for } p C R \\
T \text { vs Other Arms } \\
T \text { in } H^{+b}\end{array}$ & $\mathrm{pCR} \mathrm{HR^{- }}$ & $\begin{array}{l}\text { P-value for } p C R \\
T \text { vs Other Arms } \\
T \text { in } \mathrm{HR}^{-b}\end{array}$ & $P \mathrm{HR}^{+} \mathrm{Vs} \mathrm{HR}^{-}$ \\
\hline Arm $1(T)$ & 16/41 (39.0\%) & NA & 8/27 (29.6\%) & NA & 8/14 (57.1\%) & NA & 0.11 \\
\hline $\operatorname{Arm} 2(\mathrm{~N})$ & 14/42 (33.3\%) & 0.63 & 8/29 (27.6\%) & 1 & 6/13 (46.2\%) & 0.71 & 0.30 \\
\hline Arm 3 (T plus $N$ ) & $21 / 42(50 \%)$ & 0.22 & 7/23 (30.4\%) & 1 & 14/19 (73.7\%) & 0.46 & 0.01 \\
\hline All arms & $51 / 126(40 \%)$ & & 23/79 (29\%) & & 28/46 (61\%) & & 0.001 \\
\hline
\end{tabular}

$T$ trastuzumab, $N$ neratinib

${ }^{a} \mathrm{CMH}$ test stratified for the time period the patients were randomized to the study (prior/after the addition of arm 3 )

${ }^{\mathrm{b}}$ Fisher's exact test

[23]. Using RNA-Seq data, the 8-gene signature was used to assign each FB-7 patient to one of the three predicted trastuzumab groups (high, intermediate, and no benefit). In these benefit groups, the $\mathrm{pCR}$ rates were $75 \%, 53 \%$, and $22 \%$, respectively, when analyzed without regard to the treatment arm (Table 5). The difference between the pCR rates of the high (9/12) and no-benefit $(2 / 9)$ groups was significant $(P=0.03)$. The $\mathrm{pCR}$ rate of the combined high and intermediate benefit groups (26/44), compared to the no benefit group (2/9), was higher $(59 \%$ vs $22 \%)$ and showed a trend for significance $(P=0.056)$.

\section{RNA-Seq analysis}

Whole transcriptome RNA-Seq analysis was used to assess the gene expression profiles in pre-treatment biopsies $(n=59$ cases) and residual tumors $(n=16$ cases) (Additional file 1: Methods). As expected, the distribution of intrinsic subtypes in the FB-7 pre-treatment biopsies $(n=59)$ showed that HER2-enriched (61\%) was the most common, but other subtypes were also present: basal (11.9\%), luminal A (6.8\%), luminal B (15.3\%), and normal (5\%). A similar distribution of subtypes for HER2-enriched, basal, and luminal B were seen in a composite of five different neoadjuvant clinical trials in clinically HER2 ${ }^{+}$patients $[24,25]$. The percentage of luminal A was higher in the composite of neoadjuvant trials (24\%) compared to FB-7, in which luminal A represented only $6.8 \%$ of the patient population.

Intrinsic subtype classifications were assessed for their association with $\mathrm{pCR}$ by combining the pre-treatment samples from all three arms. The pCR rates were highest in the basal and HER2-enriched subtypes, 3/5 (60\%) and $22 / 36$ (61\%), respectively, and lowest in the luminal subtypes $3 / 9(33 \%)$ as seen elsewhere [24].

Comparison of intrinsic subtypes between the matched pre-treatment biopsies and post-treatment residual tumors revealed that $6 / 11(55 \%)$ cases converted to a normal-like subtype (Additional file 1: Figure S2). The PAMELA trial [25] similarly observed that nearly half of breast tumors became normal-like (48.9\%) after treatment with anti-HER2 therapy.

\section{Discussion}

This study showed a numerical improvement in the pCR rate in all patients treated with trastuzumab plus neratinib (50\%) compared to either the trastuzumab arm (38\%) or the neratinib arm (33\%). This study was not powered to evaluate the statistical significance between the arms but rather to assess the activity in each arm. Similar observations regarding a non-significant, numerical increase in the pCR rate in patients treated with dual anti-HER2 therapy were seen in the CALGB $40601(n=233)$ and NSABP B-41 $(n=347)$ trials, both of which included the arms with trastuzumab and the combination of lapatinib with

Table 4 NSABP FB-7: association of FCGR3A-158FN single nucleotide polymorphisms (SNP) with pathologic complete response (pCR)

\begin{tabular}{|c|c|c|c|c|c|c|}
\hline Treatment arm & $\begin{array}{l}\mathrm{pCR} \\
\text { status }\end{array}$ & $\begin{array}{l}\mathrm{F} / \mathrm{F} \\
\text { patients }\end{array}$ & $\begin{array}{l}\mathrm{pCR} \text { rate in } \mathrm{F} / \mathrm{F} \\
(\%)\end{array}$ & $\begin{array}{l}\text { VN or V/F } \\
\text { patients }\end{array}$ & $\begin{array}{l}\text { pCR rate in } \mathrm{V} N \text { or } \mathrm{V} / \mathrm{F} \\
(\%)\end{array}$ & $\begin{array}{l}\text { Compare } F / F \text { to } V / F \\
\text { and } V / F P \text { values }\end{array}$ \\
\hline \multirow[t]{2}{*}{ Trastuzumab } & No $p C R$ & 7 & \multirow{2}{*}{13} & 3 & \multirow{2}{*}{72.7} & \multirow{2}{*}{0.02} \\
\hline & $\mathrm{pCR}$ & 1 & & 8 & & \\
\hline \multirow[t]{2}{*}{ Neratinib } & No pCR & 9 & \multirow{2}{*}{25} & 6 & \multirow{2}{*}{50.0} & \multirow{2}{*}{0.4} \\
\hline & $\mathrm{pCR}$ & 3 & & 6 & & \\
\hline \multirow[t]{2}{*}{ Trastuzumab plus neratinib } & No pCR & 4 & \multirow{2}{*}{43} & 3 & \multirow{2}{*}{62.5} & \multirow{2}{*}{0.6} \\
\hline & $\mathrm{pCR}$ & 3 & & 5 & & \\
\hline \multirow[t]{2}{*}{ All arms } & No pCR & 20 & \multirow{2}{*}{25.9} & 12 & \multirow{2}{*}{61.3} & \multirow{2}{*}{0.009} \\
\hline & $\mathrm{pCR}$ & 7 & & 19 & & \\
\hline
\end{tabular}


Table 5 NSABP FB-7: pathologic complete response (pCR) rates in 8-gene signature trastuzumab benefit groups

\begin{tabular}{llllll}
\hline 8-Gene trastuzumab benefit prediction group & No. of samples & pCR rate in all arms (\%) & Trastuzumab & Neratinib & Trastuzumab plus neratinib \\
\hline No benefit & 9 & 22 & $1 / 3(33 \%)$ & $0 / 5(0 \%)$ & $1 / 1(100 \%)$ \\
Intermediate benefit & 32 & 53 & $4 / 8(50 \%)$ & $5 / 10(50 \%)$ & $8 / 14(57 \%)$ \\
Most benefit & 12 & 75 & $4 / 4(100 \%)$ & $4 / 7(57 \%)$ & $1 / 1(100 \%)$ \\
\hline
\end{tabular}

trastuzumab. However, NeoALTTO $(n=301)$, which also tested the combination of trastuzumab plus lapatinib, showed a statistically significant overall increase in the pCR rate for dual anti-HER2 therapy compared to singleagent trastuzumab. The pCR rate in the NeoALTTO trial was lower in the single anti-HER2 arm (29.5\%) than that in B-41, CALGB 40601, and NSABP FB-7 (49.4\%, 60.1\%, and $39.0 \%$, respectively). The low $\mathrm{pCR}$ rate of the singleagent arm in NeoALTTO may have contributed to the observed statistically significant increase in the pCR rate in patients treated with dual anti-HER2 therapy.

Among $\mathrm{HR}^{-}$patients, FB-7 was also consistent with four other neoadjuvant trials $[11,12,17,26]$, which showed that dual anti-HER2 therapy (trastuzumab plus pertuzumab or trastuzumab plus TKI) resulted in a numerically higher $\mathrm{pCR}$ rate. Among $\mathrm{HR}^{+}$patients, only the NeoALTTO study showed a statistically significant difference between single- and dual anti-HER2 agents (Additional file 1: Table S2). The difference between the $\mathrm{pCR}$ rates in $\mathrm{HR}^{+}(7 / 23)$ and $\mathrm{HR}^{-}(14 / 19)$ tumors reached significance in FB-7 patients treated with dual anti-HER2 therapy $P=0.01$ (Table 3 ). Taken together, these observations in neoadjuvant trials suggest that $\mathrm{HR}^{-}$patients, more so than $\mathrm{HR}^{+}$patients, may benefit from additional anti-HER therapy beyond trastuzumab.

The results for $\mathrm{HR}^{+}$patients in the neoadjuvant setting were different from those in the extended adjuvant setting. The ExteNET trial [27], which evaluated the benefit of neratinib vs placebo after trastuzumab-based adjuvant therapy in HER2-positive breast cancer, reported statistically significant fewer invasive disease-free survival (iDFS) events in the neratinib group (HR 0.73, 95\% CI $0.57-0.92, P=0.0083)$, which was the most significant in the $\mathrm{ER}^{+}$patients. Inferring results from the observations in the neoadjuvant setting and applying them to the adjuvant or extended adjuvant setting is problematic because of the differences in tumor burden, endpoints, and drug. Although the mechanisms have not been fully elucidated to account for a greater effect of neratinib in $\mathrm{HR}^{+}$patients than in those patients with $\mathrm{HR}^{-}$disease, it has been postulated that there is a bidirectional crosstalk between the estrogen receptor and the HER2 receptor signaling requiring concomitant block in both pathways for therapy efficacy. In the neoadjuvant trials, $\mathrm{HR}^{+}$patients were given hormonal therapy after anti-HER2 therapy, but in ExteNET, most $\mathrm{HR}^{+}$patients received concurrent hormonal therapy. This may partly account for the differential benefit from dual anti-HER2 therapy seen in $\mathrm{HR}^{+}$patients in the neoadjuvant and adjuvant settings. Interestingly, in NSABP B-52 (a neoadjuvant trial in HER2-positive, estrogen receptor-positive women), the concomitant treatment with trastuzumab plus pertuzumab and endocrine therapy did not increase the $\mathrm{pCR}$ rate, which may indicate that there is a unique interaction with neratinib and trastuzumab [28].

Using an identical regimen of trastuzumab plus neratinib, the I-SPY2 study reported that neratinib was superior to trastuzumab with a high degree of probability $(95 \%)$ in $\mathrm{HER}^{+} / \mathrm{HR}^{-}$patients as measured by a pCR of $56 \%$ in the neratinib group vs $33 \%$ in the trastuzumab (control) group. I-SPY2 specifically excluded patients who had $\mathrm{HR}^{+}$and low-risk tumors according to the 70gene MammaPrint assay. To determine if the FB-7 population was similar to that of I-SPY2, a subset of 45 baseline tissue samples was evaluated by the MammaPrint assay, and only 3 patients ( 2 patients in arm 1 [trastuzumab] and 1 patient in arm 2 [neratinib]) were found to be low risk, none of whom achieved a pCR. Therefore, it is unlikely that patient eligibility accounts for the discordant results. Another difference in our trial was the randomization procedure. FB-7 used a fixed randomization, whereas I-SPY2 used an adaptive randomization procedure, putting more patients in the treatment arm with a higher rate of pCR.

As expected, the major toxicity associated with neratinib in FB-7 is diarrhea. In the neratinib-alone and trastuzumab plus neratinib arms, nearly all patients experienced some diarrhea, with the occurrence of grade 3 at $31 \%$. During the study, primary prophylactic antidiarrheal therapy was instituted and the duration of intense anti-diarrheal therapy was increased from 2 to 4 weeks. These measures were somewhat effective in decreasing grade 3 occurrences. Notwithstanding, a dose reduction of neratinib was required in $38 \%$ and $52 \%$ of patients receiving single-agent neratinib or the combination, respectively. Premature discontinuation of antiHER2 regimen during the first 16 weeks of treatment occurred in $19 \%, 21 \%$, and $40 \%$ in arms 1,2 , and 3 , respectively. Although the addition of neratinib to trastuzumab failed to reach a statistically significant increase in the overall $\mathrm{pCR}$, a sizable proportion of patients did not receive the anticipated full course of therapy. Future development of neratinib will require strategies to improve the management of acute diarrheal 
toxicities. The CONTROL trial [29] suggests that budesonide, an oral, poorly absorbed steroid, or colestipol, which binds bile acids, may decrease the occurrence of grade 3 neratinib-associated diarrhea and lower the early discontinuation rate. Additional work is required to optimize the dose and schedule of neratinib alone and in combination with other agents. Moreover, the identification of a biomarker to determine whether there is a unique benefit to a TKI beyond anti-HER2 antibodies would be a clinically important advance.

Of the 11 paired tissue samples, $6(55 \%)$ converted to a normal-like subtype in the post-treatment residual tumor sample regardless of the subtype of the matched pretreatment biopsy. This conversion to a normal-like subtype was not due to a loss of tumor cellularity, because the percent of tumor cells in the residual tumor was similar to the tumor content of the primary biopsy. This conversion of the intrinsic subtype, also observed by others [25], may be due to the selection of normal-like cells that were in the tumor before treatment or due to an actual change of the subtype under the pressure of treatment. Conversion of tumors to a normal-like subtype, if prognostic of recurrence, may be important in determining further treatment of patients with residual disease.

We show that patients with at least one high-affinity FCGR3A allele (FCGR3A-158V) had a statistically significantly higher $\mathrm{pCR}$ rate than patients who were homozygous for the low-affinity allele (FCGR3A-158F) across all trastuzumab-based treatment arms. This association was expected because antibody-mediated ADCC activity is higher in patients with FCGR3A-158V/V genotypes than in patients with $\mathrm{F} / \mathrm{F}$ genotypes, probably due in part to the greater affinity of the FCyRIIIA-158V for the Fc region of an antibody than the FCyRIIIA-158F [30, 31]. The efficacy of neratinib is not dependent upon ADCC activity, and as predicted, we see no statistically significant differences in the pCR rates between genotypes in the neratinib arm. Although not statistically significant, the addition of neratinib to trastuzumab numerically improved $\mathrm{pCR}$ in $\mathrm{F} / \mathrm{F}$ patients, from 1 in 8 patients to 3 in 7 .

We realize that there are several limitations to this study, which prevent firm conclusions in both the clinical findings and the correlative analyses. The small sample size and changing landscape of treatment required several amendments to the study including the addition of a third arm as well as dropping tissue requirements. This necessitated a non-comparative statistical design for the primary endpoint of pCR. As pointed out, there was a trend toward increased $\mathrm{PCR}$ in $\mathrm{HR}^{-}$patients with dual anti-HER2 therapy relative to trastuzumab, which is consistent with other similar trials. The correlative findings, although lacking sufficient power to clearly confirm predictive biomarkers, suggest that the HR receptor status, the FCGR3A-158V/F SNP, and the 8-gene trastuzumab predictive signature may be useful markers for identifying patients less likely to achieve a pCR.

In agreement with other studies, we show herein that intrinsic subtypes are associated with $\mathrm{pCR}$ and may provide predictive value within $\mathrm{HER} 2^{+}$disease, but this has not been validated and their clinical utility is somewhat limited [24]. Intrinsic subtypes are not predictive of trastuzumab benefit in the adjuvant setting [32]. In this study, we show that the subset of patients who were predicted to receive no benefit from trastuzumab, based on the 8-gene signature in FB-7, had a pCR rate of only $22 \%$. The low pCR rate and the use of a validated signature indicating that they are unlikely to receive trastuzumab benefit suggest that this patient population would constitute a subset of patients who might benefit from additional or alternative therapies. Such an approach may be useful in the design of new clinical trials following validation of the 8-gene signature in the neoadjuvant setting. Ultimately, the goal of studying novel therapies in the neoadjuvant setting is to personalize treatment to optimize benefit and minimize potential toxicities.

\section{Conclusions}

Overall, trastuzumab plus neratinib resulted in a numerical improvement in the pCR rate (50\%) compared to either the trastuzumab arm (39\%) or the neratinib arm (33\%), with pCR for each arm being greater in the $\mathrm{HR}^{-}$ patients than $\mathrm{HR}^{+}$patients (Table 3). However, in spite of primary anti-diarrheal prophylaxis, diarrhea remains the major toxicity associated with neratinib and requires further investigation for mitigation.

Across all trastuzumab-based treatments, patients with at least one high-affinity FCGR3A allele (FCGR3A-158V) had a statistically significantly higher $\mathrm{pCR}$ rate than patients who were homozygous for the low-affinity allele (FCGR3A-158F). Neratinib, which is not dependent upon ADCC activity, showed no statistically significant differences in the pCR rates between genotypes. Finally, we show that our 8-gene signature was predictive of $\mathrm{pCR}$ and warrants further investigation in HER2-positive patients.

\section{Supplementary information}

Supplementary information accompanies this paper at https://doi.org/10. 1186/s13058-019-1196-y.

Additional file 1: Methods. Table S1. Paclitaxel, trastuzumab, and neratinib drug intensity during weekly paclitaxel phase: NSABP FB-7. Table S2. Pathologic complete response ( $p C R)$ rates in neoadjuvant HER2 ${ }^{+}$breast cancer trials: NSABP FB-7. Figure S1. MammaPrint Scores for patients enrolled in NSABP FB-7. Randomized patients were assigned a MammaPrint score based on the propriatory test from Agendia. Figure S2. Intrinsic subtypes differ in residual tumors compared to the pretreatment biopsy: NSABP FB-7. 


\section{Authors' contributions}

SAJ was the protocol officer and had substantial contributions to the conception/design of the work, acquisition/analysis/interpretation of the data, and drafting/critical revisions for the work. AR was the protocol chair and had substantial contributions to the data acquisition/patient accrual. JA had substantial contributions to the conception/design of the work, acquisition/analysis/interpretation of the data, and drafting/critical revisions for the work. JMP-G, NLV, JMO, MEC, SA, EA, SDC, and AL-C contributed to the data acquisition/patient accrual. FP was the protocol statistician and had substantial contributions to the analysis/interpretation of the data. JC had substantial contributions to the data acquisition/patient accrual and interpretation of the data. RSK, HF, CL, PCL, AS, YW, NS, and PGG had substantial contributions to the analysis/interpretation of the data. ADB had substantial contributions to the conception/design of the work. SP is the protocol pathologist and had substantial contributions to the analysis/interpretation of the data. CJA and KLP-G had substantial contributions to the conception/design of the work, analysis/interpretation of the data, and drafting/critical revisions for the work. NW is the NSABP chair/principal investigator. The research was conducted in ASST Fatebenefratelli Sacco - PO Fatebenefratelli, Milan, Italy. All authors approved the final manuscript and submission thereof.

\section{Funding}

Puma Biotechnology, Inc.

The NSABP Foundation received funding from Puma Biotechnology to conduct the clinical trial and correlative science associated with this clinical trial. No authors received any direct funding to this research but indirectly received salary support for efforts to conduct this research.

The funder played no role in the design of the study; collection, analysis, or interpretation of the data; or writing of the manuscript or submission thereof.

\section{Availability of data and materials}

The datasets during and/or analyzed during the current study will be available from the corresponding author on reasonable request and with permission of PUMA Biotechnology.

Name of the registry: ClinicalTrials.gov

Trial registration number: NCT01008150

Date of registration: November 3, 2009

\section{Ethics approval and consent to participate}

The study protocol was approved by the Institutional Review Boards of each participating institution, and all patients provided written informed consent. The study was conducted according to the Good Clinical Practice and the Declaration of Helsinki and its amendments. The authors had full control of all primary data.

\section{Consent for publication}

Not applicable.

\section{Competing interests}

Dr. Pérez-Garcia reports advisory roles with Roche/Lilly/MedSIR.

Dr. Cortes reports the following: consulting/advisor: Roche, Celgene, Cellestia, AstraZeneca, Biothera Pharmaceutical, Merus, Seattle Genetics Daiichi Sankyo, Erytech, and Athenex; honoraria: Roche, Novartis, Celgene, Eisai, Pfizer, and Samsung; research funding to institution: Roche, Ariad pharmaceuticals, AstraZeneca, Baxalta GMBH/Servier Affaires, Bayer Healthcare, Eisai, F.Hoffman-La Roche, Guardant health, Merck Sharp\&Dohme, Pfizer, Piqur Therapeutics, Puma C, and Queen Mary University of London; and stock, patents, and intellectual property: MedSIR

Dr. Di Cosimo reports the following: Speaker's Bureau: Novartis Pharma. Dr. La Verde reports the following conflicts of interest: Pfizer, Eisai, Roche, Novartis, Celldex, and TEVA.

Dr. Lucas reports stock ownership in Amgen and consulting activity with Bayer/Loxo.

All other authors declare that they have no competing interests.

\section{Author details}

${ }^{1}$ NSABP Foundation, Inc., Nova Tower 2, Two Allegheny Center - Ste 1200, Pittsburgh, PA 15212, USA. 'Centre hospitalier de l'université de Montréal, Montréal, QC, Canada. ${ }^{3}$ Cleveland Clinic, Cleveland, OH, USA. ${ }^{4}$ QuironSalud Group, IOB Institute of Oncology, Madrid, Barcelona, Spain. ${ }^{5}$ Medica Scientia
Innovation Research (MedSIR), Barcelona, Spain. ${ }^{6}$ Present address: ASST Fatebenefratelli Sacco - PO Luigi Sacco, Milan, Italy. ${ }^{7}$ ASST Fatebenefratelli Sacco - PO Fatebenefratelli, Milan, Italy. ${ }^{8}$ Roper St. Francis Healthcare, Charleston, SC, USA. ${ }^{9}$ Azienda Ospedaliera San Gerardo, Monza, Italy.

${ }^{10}$ International Drug Development Institute (IDDI), Louvain-la-Neuve, Belgium.

${ }^{11}$ Hospital Universitario, Coruña, Spain. ${ }^{12}$ Fondazione IRCCS Istituto Nazionale di Tumori, Milan, Italy. ${ }^{13}$ Department of Pathology, University of Pittsburgh School of Medicine, Pittsburgh, PA, USA. ${ }^{14}$ Severance Biomedical Science Institute and Department of Medical Oncology, Yonsei University College of Medicine, Seoul, Republic of South Korea. ${ }^{15}$ Department of Medicine, University of Florida Health, Gainsville, FL, USA. ${ }^{16}$ University of Pittsburgh, Pittsburgh, PA 15212, USA.

Received: 13 June 2019 Accepted: 4 September 2019

Published online: 03 December 2019

\section{References}

1. Slamon DJ, Clark GM, Wong SG, et al. Human breast cancer: correlation of relapse and survival with amplification of the HER-2/neu oncogene. Science. 1987;235:177-82.

2. Slamon DJ, Leyland-Jones B, Shak S, et al. Use of chemotherapy plus a monoclonal antibody against HER2 for metastatic breast cancer that overexpresses HER2. N Engl J Med. 2001;344:783-92.

3. Romond EH, Perez EA, Bryant J, et al. Trastuzumab plus adjuvant chemotherapy for operable HER2-positive breast cancer. N Engl J Med. 2005;353:1673-84.

4. Piccart-Gebhart MJ, Procter M, Leyland-Jones B, et al. Trastuzumab after adjuvant chemotherapy in HER2-positive breast cancer. N Engl J Med. 2005; 353:1659-72.

5. Slamon D, Eiermann W, Robert N, Breast Cancer International Research Group, et al. Adjuvant trastuzumab in HER2-positive breast cancer. N Engl J Med. 2011;365:1273-83.

6. Nahta R, Esteva FJ. HER2 therapy: molecular mechanisms of trastuzumab resistance. Breast Cancer Res. 2006;8:215.

7. Yarden Y, Sliwkowski MX. Untangling the ErbB signaling network. Nat Rev Mol Cell Biol. 2001;2:127-37.

8. Konecny GE, Pegram MD, Venkatesan N, et al. Activity of the dual kinase inhibitor lapatinib (GW572016) against HER-2-overexpressing and trastuzumab-treated breast cancer cells. Cancer Res. 2006;66:1630-9.

9. Rusnak DW, Lackey K, Affleck K, et al. The effects of the novel, reversible epidermal growth factor receptor/ErbB-2 tyrosine kinase inhibitor, GW2016, on the growth of human normal and tumor-derived cell lines in vitro and in vivo. Mol Cancer Ther. 2001;1:85-94.

10. Geyer CE, Forster J, Lindquist D, et al. Lapatinib plus capecitabine for HER2positive advanced breast cancer. N Engl J Med. 2006;355:2733-43.

11. Baselga J, Bradbury I, Eidtmann H, et al. Lapatinib with trastuzumab for HER2-positive early breast cancer (NeoALTTO): a randomized, open-label, multicenter, phase 3 trial. Lancet 2012;379:633-40. Erratum in: Lancet 2012; 379:616.

12. Carey LA, Berry DA, Cirrincione $C T$, et al. Molecular heterogeneity and response to neoadjuvant human epidermal growth factor receptor 2 targeting in CALGB 40601, a randomized phase III trial of paclitaxel plus trastuzumab with or without lapatinib. J Clin Oncol. 2016;34:542-9.

13. Rabindran SK, Discafani CM, Rosfjord EC, et al. Antitumor activity of HKI-272, an orally active, irreversible inhibitor of the HER-2 tyrosine kinase. Clin Cancer Res. 2004;64:3958-65.

14. Burstein HJ, Sun Y, Dirix LY, et al. Neratinib, an irreversible ErbB receptor tyrosine kinase inhibitor, in patients with advanced ErbB2-positive breast cancer. J Clin Oncol. 2010;28:1301-7.

15. Chow LW, Xu B, Gupta S, et al. Combination neratinib (HKI-272) and paclitaxel therapy in patients with HER2-positive metastatic breast cancer. Br J Cancer. 2013;108:1985-93.

16. Jankowitz RC, Abraham J, Tan AR, et al. Safety and efficacy of neratinib in combination with weekly paclitaxel and trastuzumab in women with metastatic HER2-positive breast cancer: an NSABP Foundation Research Program phase 1 study. Cancer Chemother Pharmacol. 2013;72:1205-12.

17. Gianni L, Pienkowski T, Im Y-H, et al. Efficacy and safety of neoadjuvant pertuzumab and trastuzumab in women with locally advanced, inflammatory, or early HER2-positive breast cancer (NeoSphere): a randomized multicentre, open-label, phase 2 trial. Lancet Oncol. 2012;13:25-32. 
18. Schneeweiss A, Chia S, Hickish T, et al. Pertuzumab plus trastuzumab in combination with standard neoadjuvant anthracycline-containing and anthracycline-free chemotherapy regimens in patients with HER2-positive early breast cancer: a randomized phase II cardiac safety study (TRYPHAENA). Ann Oncol. 2013;24:2278-84.

19. Wolff $\mathrm{AC}$, Hammond $\mathrm{MEH}$, Allison $\mathrm{KH}$, et al. Human epidermal growth factor receptor 2 testing in breast cancer: American Society of Clinical Oncology/ College of American Pathologists Clinical Practice Guideline Focused Update. Arch Pathol Lab Med. 2018;142:1364-82.

20. Gavin PG, Song N, Kim SR, et al. Association of polymorphisms in FCGR2A and FCGR3A with degree of trastuzumab benefit in the adjuvant treatment of ERBB2/HER2-positive breast cancer: analysis of the NSABP B-31 trial. JAMA Oncol. 2017;3:335-41.

21. Park JW, Liu MC, Yee D, et al. I-SPY2 investigators. Adaptive randomization of neratinib in early breast cancer. N Engl J Med. 2016;375:11-22.

22. Pogue-Geile KL, Kim C, Jeong J-H, et al. Predicting degree of benefit from adjuvant trastuzumab in NSABP trial B-31. J Natl Cancer Inst. 2013;105:1782-8

23. Pogue-Geile KL, Song N, Serie DJ, Thompson EA. The NSABP/NRG 8-gene signature accurately predicts degree of benefit from trastuzumab in Alliance/NCCTG N9831: validation of the 8-gene signature in an independent clinical trial. Cancer Res. 2018;78(4 Suppl):Abstr PD3-18.

24. Prat A, Pascual T, Adamo B. Intrinsic molecular subtypes of HER2+ breast cancer. Oncotarget. 2017:8:73362-3.

25. Prat, Aleix. HER2-enriched breast tumor subtype predicts response to HER2 dual blockade. Published by Kuznar, Wayne, in OncLive, Dec 08, 2016. Accessed 8-31-18: https://www.onclive.com/web-exclusives/her2-enrichedbreast-tumor-subtype-predicts-response-to-her2-dual-blockade

26. Robidoux A, Tang G, Rastogi $P$, et al. Lapatinib as a component of neoadjuvant therapy for HER2-positive operable breast cancer (NSABP protocol B-41): an open-label, randomized phase 3 trial. Lancet Oncol. 2013; 14:1183-92.

27. Martin M, Holmes FA, Ejlertsen B, et al. Neratinib after trastuzumab-based adjuvant therapy in HER2-positive breast cancer (ExteNET): 5-year analysis of a randomised, double-blind, placebo-controlled, phase 3 trial. Lancet Oncol. 2017;18:1688-700.

28. Rimawi MF F, Cecchini RS S, Rastogi $P$, et al. A phase III trial evaluating pCR in patients with HR+, HER2-positive breast cancer treated with neoadjuvant docetaxel, carboplatin, trastuzumab, and pertuzumab (TCHP) +/- estrogen deprivation: NRG Oncology/NSABP B-52. SABCS Abstr S3-06, Dec 8, 2016.

29. Barcenas $\mathrm{CH}$, Hurvitz SA, Di Palma JA, et al. Effect of prophylaxis on neratinibassociated diarrhea and tolerability in patients with HER2+ early-stage breast cancer: phase II CONTROL trial. J Clin Oncol. 2019;37(Suppl):Abstr 548. https:// www.pumabiotechnology.com/docs/June\%202,\%202019\%20-\%20ASCO\%202 019\%20-\%20Puma\%20CONTROL\%20Poster.pdf

30. Koene HR, Kleijer M, Algra J, et al. Fc gammaRIlla-158V/F polymorphism influences the binding of IgG by natural killer cell Fc gammaRIlla, independently of the Fc gammaRIIla-48L/R/H phenotype. Blood. 1997;90: 1109-14.

31. Wu J, Edberg JC, Redecha PB, et al. A novel polymorphism of FcgammaRIIla (CD16) alters receptor function and predisposes to autoimmune disease. J Clin Invest. 1997;100:1059-70.

32. Pogue-Geile KL, Song N, Jeong JH, et al. Intrinsic subtypes, PIK3CA mutation, and the degree of benefit from adjuvant trastuzumab in the NSABP B-31 trial. J Clin Oncol. 2015;33:1340-7.

\section{Publisher's Note}

Springer Nature remains neutral with regard to jurisdictional claims in published maps and institutional affiliations.

Ready to submit your research? Choose BMC and benefit from:

- fast, convenient online submission

- thorough peer review by experienced researchers in your field

- rapid publication on acceptance

- support for research data, including large and complex data types

- gold Open Access which fosters wider collaboration and increased citations

- maximum visibility for your research: over $100 \mathrm{M}$ website views per year

At BMC, research is always in progress.

Learn more biomedcentral.com/submissions 